\title{
An examination of the determinants of low muscle mass and low muscle strength in older adults in Ireland
}

\author{
E. Flanagan ${ }^{1}$, A.M. McMorrow ${ }^{1,2}$, H. Cummins ${ }^{3}$, K. McElroy ${ }^{1}$, M.J. McGowan ${ }^{4}$, S. Rafferty ${ }^{5}$, \\ B. $\operatorname{Egan}^{6}$, G. De Vito ${ }^{7}$, S.N. McCarthy ${ }^{3}$, C.A. Corish ${ }^{7}$, C.H. Murphy ${ }^{1}$ and H. Roche \\ ${ }^{1}$ Nutrigenomics Research Group, UCD Conway Institute of Biomolecular and Biomedical Research / UCD Institute of \\ Food \& Health, University College Dublin, Belfield, Dublin 4, \\ ${ }^{2}$ Department of Clinical Nutrition, St. James's Hospital, Dublin 8, \\ ${ }^{3}$ Teagasc Food Research Centre, Ashtown, Dublin 15, \\ ${ }^{4}$ Health Service Executive Community Healthcare Organisation 6, Civic Centre, Bray, Co. Wicklow, \\ ${ }^{5}$ Health Service Executive Community Healthcare Organisation 9, Nexus Building, Blanchardstown Corporate Park, \\ Dublin 15, \\ ${ }^{6}$ School of Health and Human Performance, Dublin City University, Glasnevin, Dublin 9 and \\ ${ }^{7}$ School of Public Health, Physiotherapy and Sports Science, University College Dublin, Belfield, Dublin 4
}

This abstract was awarded the student prize for best oral original communication.

The age-related loss of muscle mass and strength is associated with frailty and loss of independence ${ }^{(1)}$. The prevalence and determinants of loss of muscle mass and muscle strength have yet to be fully characterised in the context of an Irish population. Previous studies have implicated total energy, protein, vitamin D and omega 3 fatty acid intakes in the attenuation of these losses ${ }^{(2)}$. The objectives of this study were to examine the determinants of handgrip strength and skeletal muscle mass index (SMI) in a cohort of community-dwelling older adults living in Ireland.

In a cross-sectional analysis, muscle mass, strength and dietary intake was assessed 280 free-living adults aged 65 years and over (76 $\pm 8 y$ ). Muscle mass was measured using bio-electrical impedance analysis (BIA) and muscle strength using a handgrip dynamometer. Dietary intake was assessed by $24-\mathrm{h}$ recall. The prevalence of low muscle mass and low muscle strength was determined according to the EWGSOP criteria (1). Multiple linear regression was conducted to examine predictors of handgrip strength (kg) and skeletal muscle mass index (SMI; $\left.\mathrm{kg} / \mathrm{m}^{2}\right)$.

The prevalence of low muscle strength was $16 \%$ in men and $32 \%$ in women. The prevalence of low muscle mass was $23 \%$ in men and $21 \%$ in women. Multiple regression models demonstrated that age, height, SMI, vitamin D intake and gender were significant predictors of handgrip strength. The model explained $57 \%$ of the variance in handgrip from the predictors $\left(p<0 \cdot 01 ; R^{2}=0 \cdot 57\right)$. Body mass, age, gender and average energy intake were predictors of SMI $\left(\mathrm{p}<0.01 ; \mathrm{R}^{2}=0.53\right)$.

Our data support previous work demonstrating that age and gender are important predictors of muscle mass and strength in older adults. Further work is required to elucidate the role of nutrient intakes in the development and progression of age-related muscle mass and strength loss.

This work was supported by funding from the Department of Agriculture, Food and the Marine and Health Research Board through the Joint Programming Initiative - A Healthy Diet for a Healthy Life (JPI HDHL) Knowledge Hub on Malnutrition in the Elderly (MaNuEL).

1. Cruz-Jentoft A, Baeyens JP, Bauer JM, Boirie Y, Cederholm T, Landi F, et al. (2010) Sarcopenia: European consensus on definition and diagnosis. Age and ageing. 39(4), 412-23.

2. Rondanelli M, Faliva M, Monteferrario F, Peroni G, Repaci E, Allieri F, et al. (2015) Novel Insights on Nutrient Management of Sarcopenia in Elderly. BioMed Research International. 2015:524948. 